\title{
MedienPädagogik
}

Zeitschrift für Theorie und Praxis der Medienbildung

\section{Visualisierung und Didaktisierung digitaler Archivbestände}

\section{Perspektiven zur Gestaltung offener Lernräume für historisches Lernen}

Katharina Gallner-Holzmann

\section{Zusammenfassung}

Archive stellen einen wichtigen Ort für historisches Lernen dar. Die zunehmende Digitalisierung ihrer Sammlungen erleichtert den Zugang für ein diverses Publikum in ganz unterschiedlichen Bildungssituationen. Didaktische Zugänge zu diesen Sammlungen gibt es allerdings derzeit wenige. Die Quellenorientierung in der Geschichtsdidaktik, archivpädagogische Konzepte und mediendidaktische Zugänge eröffnen eine Schnittmenge, die in online Archivzugängen bisher weitgehend ungenutzt bleibt. Dieser Beitrag präsentiert Überlegungen zur Gestaltung digitaler Archive, die das Potential eben dieser Überschneidungen nutzt. Als zentrales Element wird die Quellenarbeit aus verschiedenen Perspektiven beleuchtet und ein Beispiel aus dem DigiVis-Projekt an der Universität Innsbruck vorgestellt. 


\title{
Visualizing and educationalization of digital archives. Perspectives on the design of open learning environments for historical learning
}

\begin{abstract}
Archives are important places for historical education. Digitizing their collections broadens the possibilities of public users to enter archives for various educational purposes. But only few digitized archives consider didactics in their design. The intersection of historical didactics, archive pedagogy and media pedagogy offers a potential for online archives yet to be explored. This article is going to discuss the possibilities of these intersections. The reflection of various sources being an important element of all three concepts is examined and an example for the implementation of these perspectives resulting of the DigiVis Project at the University of Innsbruck is going to be discussed.
\end{abstract}

\section{Archive und das Internet - Was bisher geschah...}

Zahlreiche archivarische Tagungen und Sammelbände der letzten zehn Jahre setzten sich mit den Auswirkungen der Digitalisierung auf das Archivwesen auseinander. Beispielsweise standen die archivwissenschaftlichen Kolloquien an der Archivschule Marburg unter den Titeln «Digitale Registraturen - digitale Archivierung. Pragmatische Lösung für kleinere und mittlere Archive?» (2011), «Digitalisierung im Archiv. Neue Wege der Bereitstellung des Archivguts» (2013), "Born Digital in the Cloud: Challenges and Solutions» (2016). Dabei wird deutlich, dass sowohl die zunehmende Digitalisierung bereits bestehender analoger Archivbestände als auch die Archivierung digitaler Sammlungen eine Herausforderung für das Archivwesen darstellen (vgl. Archivschule Marburg o.J.). Das erste Heft des Archivars 2018 titelte «Archive 2.0» und diskutierte vor allem den Auftritt von Archiven in unterschiedlichen digitalen Medien. Neben Archivhomepages und archivalischem Bloggen wurden auch die Möglichkeiten einer Nutzung der sozialen Medien diskutiert (vgl. dazu Odendahl 2018; Unger 2018; Wolf 2018; Rönz 2018; van Kan 2018; Diener Steackling 2018). Immer wieder wird dabei angemerkt, dass viele Digitalisierungsvorhaben das Ziel verfolgen, 
den Arbeitsaufwand für Archivare zu verringern, indem die Zugänglichkeit der Archive bereits online gegeben ist. Glaunert (2015) argumentiert allerdings, dass genau das nicht der Fall ist. Die Digitalisierung ganzer Bestände ist ein teures Unterfangen, die digitale Archivierung und die Betreuung von Besuchern und Besucherinnen auf den digitalen Archivseiten erfordert zusätzliche Ressourcen, da es eben nicht ausreicht, digitale Sammlungen auf eine Plattform hochzuladen (vgl. Glaunert 2015, 4-5).

Diesen Umstand veranschaulichen zwei Szenarien. Das erste schildert Mitchell Whitelaw in seinem Plädoyer für Generous Interfaces:

«Imagine yourself outside an art gallery in a far-off city, with a collection you don't know well. You enter the building to find a small, drab lobby with an attendant at a desk. The attendant asks you to enter your query on a small slip of paper. Not knowing the collection, and not seeking anything in particular, you write down something arbitrary and pass it over. The attendant disappears for a moment before returning with a line of artworks sitting on trollies. These are paraded, ten at a time, through the lobby.» (Whitelaw 2015, 1)

Die erste Begegnung mit einer digitalen Sammlung, die man nicht kennt und an die man keine gezielte Frage stellt, sondern die man neugierig erkunden möchte, gestaltet sich in dieser Szene als einschüchternd und frustrierend.

Mitchel Whitelaw plädiert daher für ein Generous Interface, das den User nicht mit einer Suchmaske allein lässt, sondern ihm vielzählige andere Wege in ein Archiv ermöglicht. Whitelaw $(2013,7)$ formuliert dazu vier zentrale Prinzipien, die ein digitaler Archivzugang erfüllen sollte:

- Show first. Don't ask

- Provide rich overviews

- Provide context

- Show high quality primary content

Eine digitale Sammlung sollte also nicht ausschliesslich über eine Suchmaske zu erschliessen sein. Grund dafür ist nicht nur die oben geschilderte Schwierigkeit, den richtigen Suchbegriff zu finden. Vielmehr argumentiert Whitelaw mit Drucker (2013), die kritisiert, dass Interfaces 
oft nach Grundsätzen des Information Retrieval und der Human-ComputerInteractions gestaltet werden. So werden wesentliche Prinzipien der Humanities, wie Ablenkung oder Flow-Erleben, ausgeschlossen oder erschwert (vgl. Drucker 2013). Whitelaw möchte mit dem Prinzip der Generous Interfaces ein anderes Angebot gestalten. Aus der Visualisierung und dem Einstieg ins Interface sollte ersichtlich sein, wie sich die Sammlung zusammensetzt und welche Komplexität beziehungsweise Querverbindungen in einer Sammlung vorhanden sind. Jedes dargestellte Artefakt, aber auch die Sammlung selbst, sollten in ihrem Kontext innerhalb des Archivs, beziehungsweise der Forschungslandschaft verortet sein. Ausserdem sollte der Zugang zu den Primärquellen immer möglich bleiben (vgl. Whitelaw 2013, 7).

Als Beispiele stellt Whitelaw von ihm gestaltete Archivzugänge vor: Im experimentellen Web Interface zur Manly Local Studies Image Library arbeitet Whitelaw mit ungefähr 7000 Bilddokumenten, die das Leben zwischen 1800 und 1990 zeigen. Der Bildbestand lässt sich nach Titeln, also Themengruppen, wie beispielsweise «Uniform» oder «Pupils» bis hin zu «Queen» sortieren, wobei die Grösse und Position des angezeigten Beispielbildes, das automatisch generiert wird und wechselt, von der Zahl der Quellen in der Themengruppe bestimmt wird. Je grösser der Bestand, desto weiter oben und grösser wird das Beispielbild angezeigt (siehe Abb 1). 


\section{MedienPädagogik}

Zeitschrift für Theorie und Praxis der Medienbildung

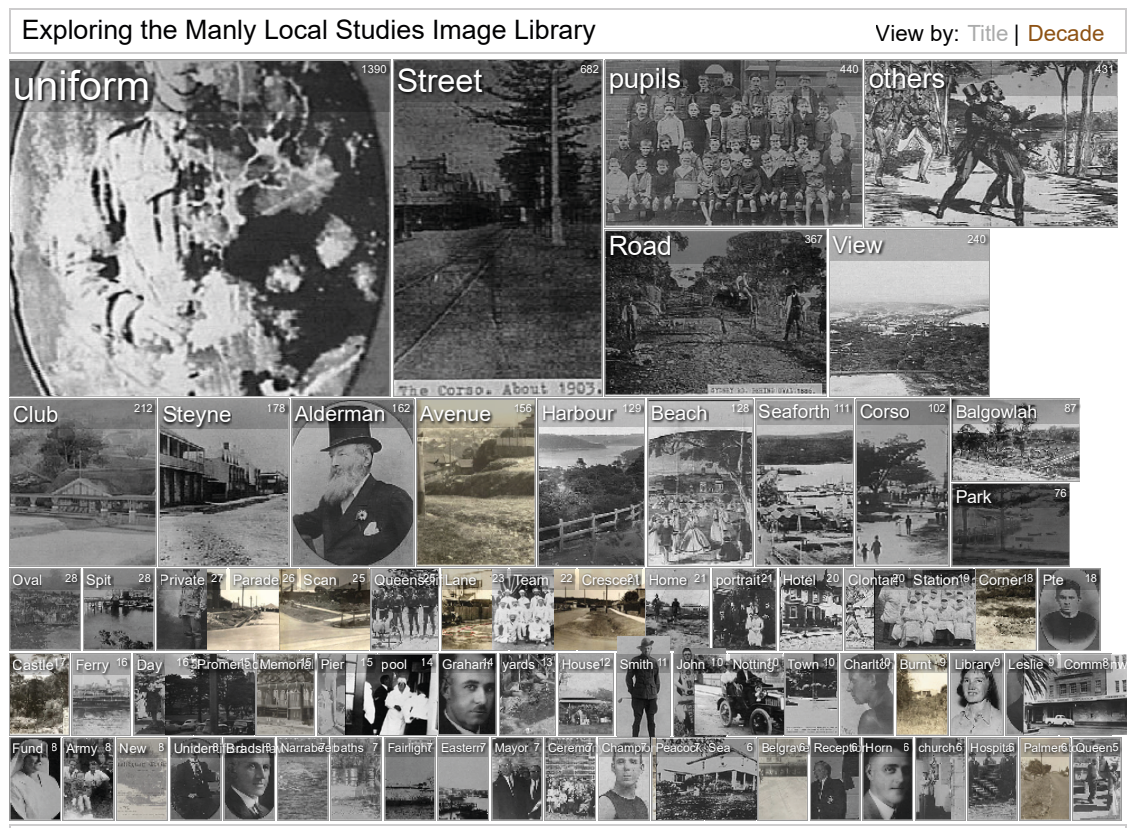

About this project | Images are copyright Manly Library, used with permission.

Abb. 1.: Screenshot des Generous Interface der Manly Image Library (Whitelaw o.J.: http://mtchl.net/manlyimages/explore.html\#title) sortiert nach Themen.

Ausserdem kann der Bestand sortiert nach Jahrzehnten angezeigt werden. Auch hier werden die Beispielbilder jener Jahrzehnte grösser angezeigt, aus welchen ein grösserer Bildbestand verfügbar ist (siehe Abb 2). 


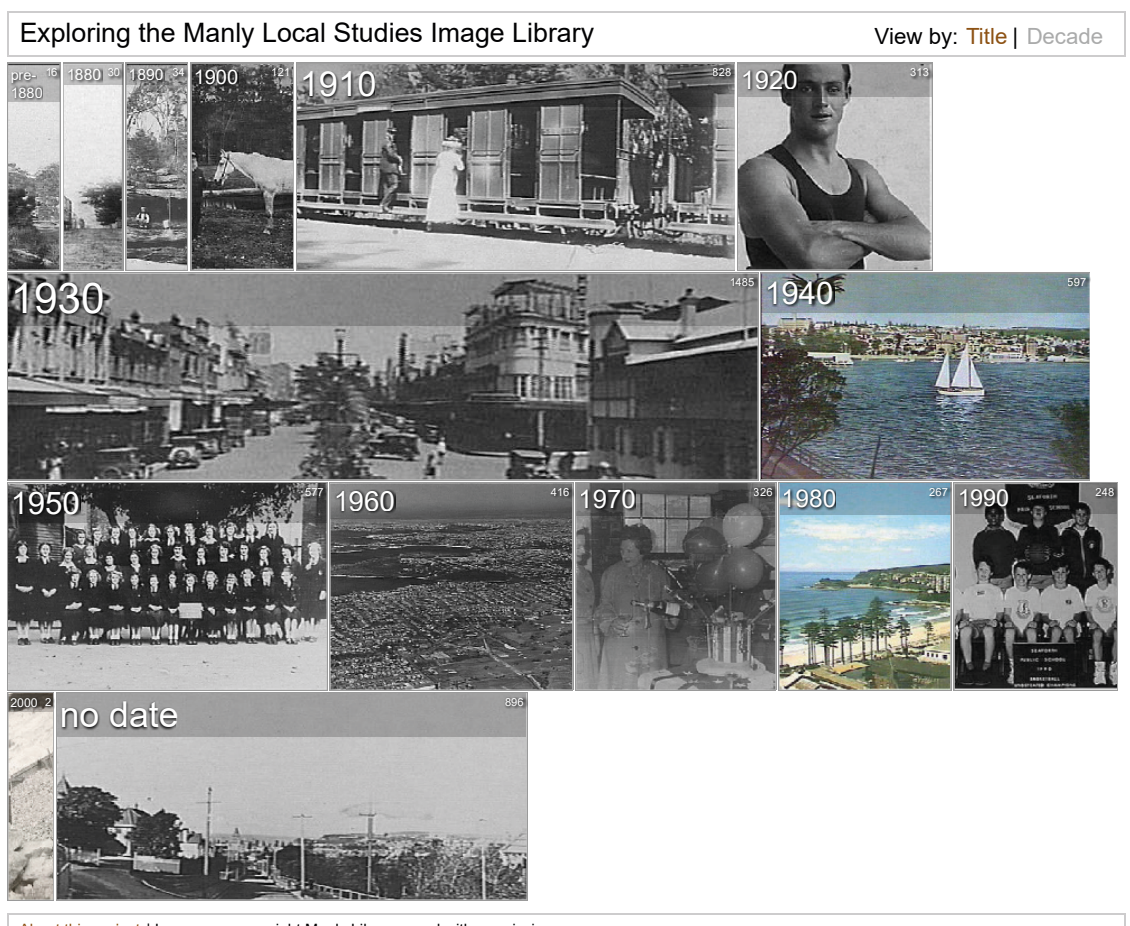

About this project | Images are copyright Manly Library, used with permission.

Abb. 2.: Screenshot des Generous Interface der Manly Image Library (Whitelaw o.J.: http://mtchl.net/manlyimages/explore.html\#decade) sortiert nach Jahrzehnten.

Bei der Auswahl eines Themenbereiches beziehungsweise eines Jahrzehnts erscheint jeweils ein Zeitstreifen, auf dem die einzelnen Bilder chronologisch abgebildet werden und von einer kurzen Bildunterschrift erklärt werden (siehe Abb. 3). 


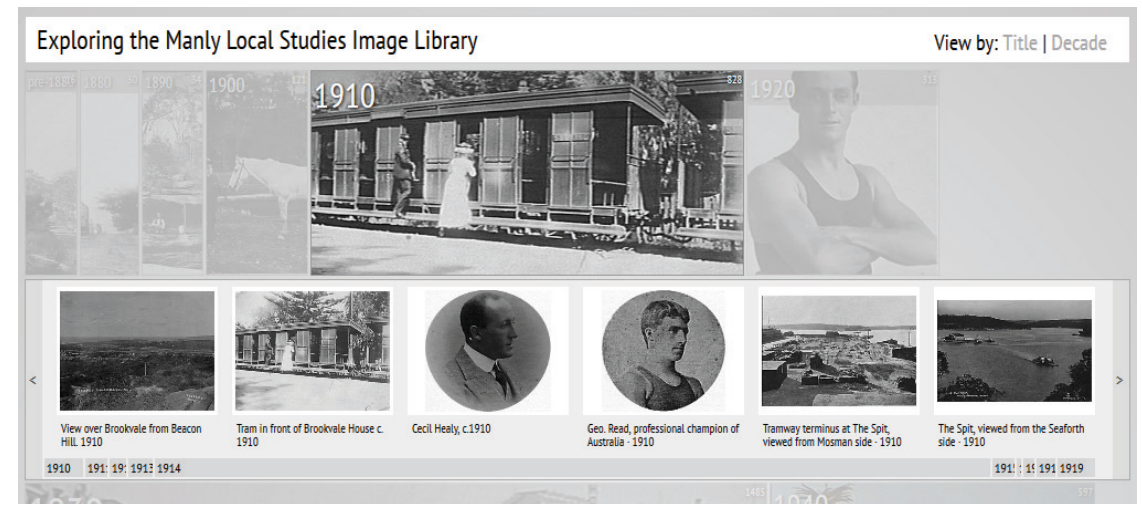

Abb. 3.: Screenshot Generous Interface der Manly Image Library (Whitelaw o.J.: http://mtchl.net/manlyimages/explore.html\#decade) nach Auswahl eines Jahrzehnts.

Whitelaw argumentiert, dass so ein einladender Zugang zum Archiv ermöglicht wird, der zur explorierenden Bewegung durch die Archive einlädt (vgl Whitelaw o.J.).

Wichtig ist Whitelaw ausserdem, dass die Darstellung der Sammlung wenig bis gar keinen zusätzlichen Kontext verleiht (vgl. Whitelaw 2015, 5; 2013 , 7). Fraglich bleibt, ob ein solcher Zugang überhaupt möglich ist. Allein durch die Zuordnung der Bilder zu Themen wird der Kontext möglicherweise eingeschränkt oder gar verändert. Whitelaw beschäftigt sich darüber hinaus nicht mit Archiven, deren Bestand überwiegend textbasiert ist und für deren Visualisierung Scans der Originaldokumente keine vielversprechende Basis darstellen.

Glaunert (2015) sieht eine weitere Herausforderung: In seinem Eröffnungsvortrag zum Brandenburgischen Archivtag 2015 beschreibt er ein weiteres Szenario, das verdeutlicht, dass eine umfassende Digitalisierung der Archivbestände ohne eine weitere Betreuung und Erschliessung schlichtweg niemanden nutzen würde:

«Es wäre also der gleiche Effekt, als würde man einen Benutzer in ein Archivmagazin stellen und ihm sagen. (Finde, was du suchst, alles ist beschriftet und geordnet. Viel Erfolg!)» (Glaunert 2015, 5) 
Diese Problematik wird auch durch Whitelaws Archivzugang nicht adressiert. Besonders bei grossen Archivbeständen kann das «Betreten» des digitalen Archivraums eine Herausforderung und schlimmstenfalls eine Überforderung darstellen. Insbesondere wenn das Hintergrundwissen zu einem bestimmten Bestand fehlt, kann auch ein erkundendes Bewegen durch Digitalisate ohne Orientierungshilfen, die über Themen und Jahreszuordnungen hinausgehen, frustrierend sein.

Glaunert sieht im Gegensatz zu Generous Interfaces die Volltextrecherche als Voraussetzung für eine sinnvolle Nutzbarkeit eines digitalen Archivs. Diese stellt aber aus technischer Perspektive in Bezug auf handschriftliche Artefakte nach wie vor eine grosse Herausforderung dar (vgl. Glaunert 2015, 5).

Obwohl in der Zwischenzeit grosse Digitalisierungsbemühungen angestellt wurden und allein über die Europeana mehr als 50 Millionen digitalisierte Objekte zugänglich sind («Europeana» o.J.), ist der Zugang zu digitalen Sammlungen nach wie vor oft nicht intuitiv ${ }^{1}$. Europeana und Manly Images sind Beispiele, wie didaktisch aufbereitete Archivseiten aussehen könnten. Die meisten Homepages und Websites von Archiven enthalten allerdings lediglich Informationen zu Sammlungen, zum Archivbesuch, Kontaktdaten und Öffnungszeiten und immer wieder auch beispielhaft einzelne Digitalisate. Digital zugängliche Sammlungen, wie das österreichische Zeitungsarchiv ANNO, sind noch dabei die Volltextsuche auszuweiten, auch wenn mit einer Abdeckung von 90\% bereits einiges passiert ist (Anno Historische Zeitungen und Zeitschriften o.J.).

Es gibt unzählige Möglichkeiten, die Archiven durch digitale Technik offen stehen und die derzeit noch nicht genutzt werden, beziehungsweise noch nicht nutzbar sind. Genannt seien hier beispielhaft Augmented Reality Szenarien, wie die automatische Transkription von gescannten Aktenseiten am Smartphone (vgl. Glaunert 2015, 9). Dabei scheint vor allem auch der Zugang zu digitalisierten Archivbeständen nach wie vor eine grundlegende Herausforderung darzustellen.

1 Das gilt sowohl für Forschende, die nach wie vor immer wieder mit unübersichtlichen Webseiten mit nicht nutzerfreundlicher Suchfunktion konfrontiert sind, als auch ganz besonders für interessierte Nutzende ohne wissenschaftliche Vorbildung. 
Kate Theimer plädiert in ihrem Vortrag im Rahmen der Konferenzreihe «Offene Archive» mit dem Schwerpunkt «Archive im Web 2.0» 2018 für ein neues Verständnis von einem Archiv als Ort «an dem man willkommen ist und [an dem] 〈Dinge passieren〉》 (Theimer 2018, 5). Dabei soll ein Archiv nicht durch einzelne kuratierte Wege in die Thematik einführen, wie das beispielsweise Museen machen, sondern im Sinne eines offenen Hypertextes (vgl. Krameritsch 2007, 152-53) eigene Erkundungen und Vernetzungen, sowohl durch Forschende und Interessierte ermöglichen und anregen.

Hier scheinen auch Überlegungen zur Berücksichtigung von postmodernen Lebensformen und ihrem Einfluss auf die Wahrnehmung von (digitalen) Bildungs- und Lernräumen, wie sie Nina Grünberger anstellt, interessant. Archive scheinen als Informationsräume nicht zuletzt für Flaneure geeignet zu sein. Ein digitales Archiv kann zum Verweilen anregen und zu vertiefenden Auseinandersetzung mit seinen Digitalisaten einladen (vgl. Grünberger 2014, 64).

\section{Potentiale für historisches Lernen in der Schnitt- menge von Medienpädagogik und Archivpädagogik}

Archivpädagogik umfasst in erster Linie die Praxis vermittelnder Aktionen in unterschiedlichen Archiven. Aufgrund enger Beziehungen der Archive zur Geschichte und einer fehlenden eigenen Archivdidaktik (vgl. Aspelmeier 2019, 106) stehen diese Aktionen oft im Zusammenhang mit geschichtsdidaktischen Zugängen, Methoden und Kompetenzen. Daher soll in diesem Kapitel zunächst das Verhältnis von Geschichtsdidaktik und Medien skizziert werden, bevor die Archivpädagogik als Teil historischen Lernens vorgestellt wird und schliesslich die Potentiale der Mediendidaktik für die archivalische Vermittlung gezeigt werden.

\subsection{Geschichtsdidaktik und Medien}

Auch geschichtsdidaktische Zugänge befassen sich zunehmend mit der Digitalisierung. Standen zu Beginn Überlegungen zum Umgang mit audiovisuellen Quellen und Darstellungen im Zentrum des Interesses, wurden ab 2004/5 zunehmend auch das Internet und veränderte 
Nutzungszusammenhänge berücksichtigt. Der immer stärker auch produzierende Zugang zu Medien führte zur Erweiterung vorhandener Werkzeuge, Arbeitsmethoden und Gegenstände in Bezug auf das Lehren und Lernen von Geschichte. Die Entwicklungen gingen dabei zunächst von Lehrenden aus, die ihre Erfahrungen reflektieren und zur fachwissenschaftlichen Diskussion stellen wollten. 2017 veröffentlichen Bernsen und Kerber schliesslich einen Sammelband, in dem sie die bisherigen Zugänge der Geschichtsdidaktik zusammenfassen und exemplarisch darstellen. Sie stellen dabei vor allem auch die Frage, welche Beiträge die Geschichtsdidaktik zur generellen und zur domänenspezifischen Medienbildung leisten kann (vgl. Bernsen und Kerber 2017, 13-16). Zentral ist dabei, dass die Vergangenheit selbst nicht zugänglich ist, sondern Informationen aus der Geschichte medial überliefert werden. Quellensammlungen, wie Dokumente, bildliche und dingliche Quellen sind Zeugnisse der Vergangenheit, die durch Verweise oder Darstellungen in zahlreichen Medien, in Biografien, Erzählungen, Filmen, Romanen aber auch Gedenktagen und Tageszeitungen zu einer übergeordneten Narration und Geschichtsdarstellung beitragen. Eine ganz zentrale Qualifikation des historischen Lernens ist daher der kompetente Umgang mit diesen Quellen und Medien (von Borries 2008, 100).

Bernsen (2017) argumentiert, dass historisches Lernen immer mit und durch Medien passiert und daher den kritischen Umgang mit Medien fördert (vgl. Bernsen 2017, S. 38). Bounin und Näpel (2015) zählen Geschichte also zu den Leitfächern bei der Vermittlung von Medienkompetenz (Bounin 2015; Näpel 2015), die hier eben nicht im Alltagsverständnis als technische Fertigkeiten wie Computer- oder Softwaretrainings, sondern im Sinne einer aktiven, reflektierten Auseinandersetzung mit Medientechniken, medialen Inhalten und historisch-medialen Konstellationen verstanden werden kann. Durch die notwendige historische Einbettung der Quelle wird darüber hinaus die Bedingung der Produktion und nicht zuletzt auch ihre Verbreitung beleuchtet (vgl. Kerres 2018, Kap. 2.3.1).

Archive sind je nach Bestand Verwahrer eben dieser Medien - von primären historischen Quellen, aber auch von unterschiedlichen Darstellungen von Geschichte. Durch eine mediale Gestaltung der Archive im Internet werden sie ausserdem selbst zu einer Darstellung von Geschichte. 
Daher scheint es bedeutend, geschichtsdidaktische Zugänge zu historischem Lernen mit Medien zu berücksichtigen und diese Doppelrolle, die ein digitales Archiv dabei einnimmt, auch kritisch zu hinterfragen.

Durch die Auswahl und die Darstellung der einzelnen Quellen und den Kontext, der durch eine Strukturierung oder Schlagworte vorgenommen wird, wird Geschichte nicht nur anhand primärer Quellen gezeigt, sondern auch hergestellt.

Whitelaws Versuch eines möglichst unkuratierten und unmittelbaren Zugang zu den Quellen (vgl. Whitelaw 2015, 3) erscheint unter diesen Gesichtspunkten und insbesondere auch in seinen Vorschlägen schwierig. Durch die Strukturierung und die Zuweisung in ein Themenfeld wird den Bildern bereits ein Kontext zugeschrieben, der ganz drastische Auswirkungen auf die Interpretation einer Quelle haben könnte.

\subsection{Archivpädagogik und historisches Lernen}

Erste Veröffentlichungen von archivarischen Akten gab es im Zuge der Aufklärung im 18. Jahrhundert. Sie waren nicht zuletzt eine Möglichkeit, um Missstände aufzuzeigen (vgl. Lange, Lux, und Mayer 2004, 16-17). Im Zuge der Französischen Revolution wurde in Frankreich die Nutzung von Archiven für jeden Bürger gesetzlich festgelegt (vgl. Lange, Lux, und Mayer 2004, 26). Wie bereits der 1794 in Paris gesetzlich garantierte Zugang zu den Archiven, ermöglicht auch das österreichische Archivnutzungsrecht einerseits juristische Auskünfte und andererseits historischen, künstlerischen und wissenschaftlichen Zugang zu Archiven (vgl. Bundesministerium für Digitalisierung und Wirtschaftsstandort 2000; Lange, Lux, und Mayer 2004, 26).

«§ 9. (1) Jedermann ist berechtigt, gemäß \& 8 freigegebenes Archivgut nach Maßgabe dieses Bundesgesetzes und im Rahmen der Benutzungsordnung ( $\$$ 10) des betreffenden Archivs des Bundes zu amtlichen, wissenschaftlichen oder publizistischen Zwecken sowie zur Wahrnehmung berechtigter persönlicher Belange zu nutzen.» (Bundesministerium für Digitalisierung und Wirtschaftsstandort 2000) 
Um dieses Recht zu nutzen, braucht es allerdings Fertigkeiten, denn ein Archiv stellt keine fertigen Interpretationszusammenhänge und Narrationen dar, wie etwa eine Museumsausstellung. Diese Fähigkeiten sollen durch archivpädagogische Angebote entwickelt werden.

Zur Herausbildung einer Archivpädagogik musste sich allerdings das Verhältnis von Geschichtsunterricht zu Quellen und des Archivs zur Schule entwickeln. Bereits Mitte des 19. Jahrhundert gab es seitens britischer Schulinspektoren die Empfehlung, originale historische Quellen in den Unterricht miteinzubeziehen und Ende des 19. Jahrhunderts arbeiteten Schulen und Archive in Deutschland und Belgien zusammen. Um 1950 etablierte sich in Frankreich der service educatif, der die Zusammenarbeit von Schulen und Archiven institutionalisierte. Ein Lehrer, der an das Archiv abgeordnet wird, ermöglicht eine personelle Verbindung zwischen den Institutionen und ergänzt den schulischen Unterricht durch archivalische Angebote, die an den Lehrstoff angepasst sind. Dabei kommen insbesondere Quellen aus der regionalen Geschichte zum Einsatz (vgl. Lange, Lux, und Mayer 2004, 26-27). Während in Grossbritannien, Frankreich und den Niederlanden verschiedene Archive umfangreiche Angebote, die an Altersgruppen und Lehrpläne angepasst sind, gestalten, stehen viele europäische Archive 2004 noch am Anfang der archivpädagogischen historischen Bildungsarbeit (vgl. Lange, Lux, und Mayer 2004, 33-35). 15 Jahre später kritisiert Jens Aspelmeier, dass die Entwicklung einer Archivdidaktik noch immer aussteht, Archive ihre Rolle als Kultur- und Bildungsinstitutionen nach wie vor nicht profiliert haben und eine systemische Entwicklung der Zusammenarbeit von Archiven mit Bildungseinrichtungen noch nicht fortgeschritten zu sein scheint (vgl. Aspelmeier 2019, 106-7).

Dabei ist Archivpädagogik mehr als die Zusammenarbeit von Archiven mit Schulen und insbesondere Geschichtslehrerinnen und -lehrern. So veranstalteten beispielsweise die Speyrer Archive ein Projekt, in dem ältere Personen ermuntert wurden, ihre Lebenserfahrungen in der Kriegs- und Nachkriegszeit für Archive niederzuschreiben (vgl. Stüber 2003, 205) und so ein Verständnis für die Arbeit von Archiven zu entwickeln.

Das Zielpublikum der archivpädagogischen Arbeit ist also divers und vielfältig sind auch die thematischen Schwerpunkte der einzelnen Archive und ihrer pädagogischen Angebote (vgl. Schaller 2019, 102). Dennoch 
bewegen sich archivpädagogische Konzepte insbesondere im Umfeld historisch politischer Bildung. Einen zentralen Anspruch formuliert dabei Annkatrin Schaller: durch die Arbeit mit den originalen Quellen kann gelungene Archivpädagogik Schülerinnen und Schüler die Erfahrung vermitteln, dass Geschichte gemacht wird (vgl. 2019, 102). Das Ziel ist also die Vermittlung eines kritischen Geschichtsbewusstseins und die Schulung von Medien- und Urteilskompetenz (Schaller 2019, 103) anhand des Zugangs zu Originalquellen als zentrale Gegenstände der Geschichte. So kommt dem Archiv die Aufgabe zu, die Bedeutung des Originals/des Echten zu vermitteln (vgl. Aspelmeier 2019, 104).

Archivpädagogik adressiert also ganz stark die Quellenorientierung in der Geschichtsdidaktik. Das Ziel der archivpädagogischen Arbeit ist es, Geschichte sinnlich und persönlich erfahrbar zu machen, Geschichte soll als offen, multiperspektivisch und manchmal auch widersprüchlich kennengelernt werden und die Erfahrung, dass Geschichte etwas Nahes, beispielsweise räumlich oder biografisch, und gleichzeitig Fernes, meist zeitlich, sein kann, soll ermöglicht werden (vgl. Lange, Lux, und Mayer 2004, 47-48).

Durch forschendes Lernen im Archiv wird auch die historische Fragekompetenz geschult, muss doch zunächst eine Fragestellung gefunden werden, nach der eine Auswahl von Quellen getroffen werden kann. Die Analyse der Archivalien erfordert Methodenkompetenz. Das Erfahren von Geschichte als etwas, das uns über Quellen zugänglich ist und durch Geschichtsdarstellungen, wie beispielsweise Schulbücher, vermittelt wird, fördert die historische Reflexionskompetenz. In der Arbeit mit Archiven wird für den Lernenden spürbar, wie die Plausibilität von Geschichtsdarstellungen überprüft werden kann (vgl. Lange, Lux, und Mayer 2004, 4849).

Dabei bietet das Archiv über das Fach hinausreichende Erkenntnismöglichkeiten insbesondere auch als Ort des lebenslangen Lernens. Identitätsbezogene Formen der Archivarbeit, wie beispielsweise die Erforschung der Familiengeschichte, können auch ausserhalb des schulischen beziehungsweise institutionellen Lernens auf Geschichte neugierig machen (vgl. Aspelmeier 2019, 106). 
Am «Rohmaterial der Geschichte» sollen Archive in ihren pädagogischen Angeboten zentrale Aspekte wie die Perspektivität der Quellen, Lücken der Überlieferung und Selektivität des Zugriffs behandeln. Dass im Geschichtsunterricht Quellen oft selbst medial vermittelt werden und die Quellenarbeit zwar in der modernen Geschichtsdidaktik einen zentralen Aspekt darstellt, im Unterrichtssetting dennoch nicht ausreichend erfahrbar gemacht werden kann, unterstützt den Anspruch an den Lernort Archiv. Jens Aspelmeier plädiert für die Arbeit an der nicht aufbereiteten Quelle, die Fragen zu Inhalt, Gattungsspezifik einer Quelle und Überlieferungsproblematiken selbst erkennbar machen. Es wird eine vertiefte Auseinandersetzung mit der Quelle, insbesondere in Abgrenzung zu Geschichtsdarstellungen möglich. Dafür ist der Besuch im Archiv sicher besser geeignet als eine Quellenabbildung im Schulbuch (vgl. 2019, 106).

Neben der Zusammenarbeit mit Schulen werden dazu auch Quellenmaterialien mit didaktischem Apparat herausgegeben. Die Möglichkeit auch online pädagogische Angebote bereitzustellen, nutzen bisher nur wenige Archive (vgl. Schaller 2019, 103). Vorhandene Angebote reichen von Lernprogrammen zu historischen Inhalten bis zu verschiedenen Lehrgängen zur Quellenarbeit sowie zum Lesen alter Handschriften (beispielsweise: https://www.adfontes.uzh.ch/300o/training).

Dabei kann aber auch der Zugriff auf eine gut aufbereitete digitalisierte Quellensammlung bereits eine Erweiterung der Darstellungsmöglichkeiten eines Schulbuchs zugänglich machen: Quellen können aus einem wesentlich grösseren zugreifbaren Korpus mit oder ohne Unterstützung einer Lehrperson ausgewählt werden. Ein 3D-Scan kann digital unterschiedliche Perspektiven auf ein Objekt ermöglichen und durch unterschiedliche digitale Tools kann die Erschliessung eines Dokuments, beispielsweise durch OCR-Scans und Transkriptionen von handschriftlichen Quellen erweitert werden. So können Lernende sich durchaus durch unterschiedliche gescannte Originaldokumente bewegen und im Sinne eines explorierenden und forschenden Lernens einen anderen Zugang zu Geschichte erfahren als im darstellenden Überblickstext eines Schulbuchs oder Sachbuchs, der oft durch die Abbildung einer exemplarischen Quelle lediglich unterstützt wird. 
Je nach Schwerpunkt des Archivs können anhand von typischen Digitalisaten Methoden gezeigt und angeleitet werden, die den historischen Umgang mit Quellen unterschiedlichster Art vermitteln.

Die mediale Vermittlung des gescannten Originals sollte dabei in Unterrichtsgesprächen oder bereits auf der Archivseite selbst immer wieder thematisiert und reflektiert werden.

\subsection{Mediendidaktik und der Zugang zu Archiven}

Parallel zur verstärkten Hinwendung der Geschichtsdidaktik zur Quelle und der verstärkten Öffnung und Nutzung der Archive rückte in der zweiten Hälfte des 20. Jahrhunderts in der Medienpädagogik die Handlungsorientierung der Mediennutzenden ins Zentrum (vgl. Kerres 2018, Kap. 2.2). Anstatt Rezipienten und Produzenten streng zu unterscheiden, stellte sich in der Medienpädagogik die Frage, wie der kompetente Umgang mit Medien erlernt werden kann und wie sich Menschen in ihrer medialen Umwelt zurechtfinden. Ganz zentral für die Arbeit mit Archiven erscheint dabei der Sachbezug, der die Teilhabe an kulturellem Wissen, das über Medien zugänglich ist, ermöglichen soll (vgl. Kerres 2018, Kap. 2.2).

Dieses kulturelle Wissen wird nicht zuletzt auch in Archiven aufbewahrt und aufbereitet.

Die Handlungsorientierung in der Medienpädagogik hat Konsequenzen für die Gestaltung digitaler Umwelten, die den Zugang zu Wissen eröffnen, die menschliche Entwicklung fördern und darüber hinaus Kommunikation anregen sollten (vgl. Kerres 2018, Kap. 2).

In der Gestaltung eines digitalen Lernraumes in der Schnittmenge von historischem Lernen und Medienbildung erscheint dieser Anspruch aus mehreren Gründen interessant: einerseits wohnt der Auseinandersetzung mit Quellen ein Moment zur Ausbildung von Medienkompetenz inne und andererseits sollte die mediale Darstellung der Quelle und die digitale Lernumgebung Archiv nicht nur in ihrer didaktischen, sondern auch in ihrer medialen Gestaltung reflektiert werden.

Das bedeutet, dass mediendidaktische Überlegungen, die das Lernpotential der Lernenden berücksichtigen, am Beginn des Gestaltungsprozesses stehen, während die Re-Konstruktion beziehungsweise Re-Konstruktionsmöglichkeiten der Geschichte in der Auswahl und Darstellung der 
Quellen sowie die Möglichkeiten mit ihnen zu arbeiten, mitgedacht werden müssen.

Eine gelungene Lernumgebung ermöglicht es also, nicht nur historische Kompetenzen zu vermitteln, sondern spricht auch zentrale Aspekte der Medienbildung an. Je nach Archivbestand können in einem mehr oder weniger engen Medienverständnis durch handlungsorientiertes historisches Lernen im Archiv auch Arbeitsbereiche der Medienbildung nach Tulodziecki (1998) angesprochen werden:

- Medienangebote sinnvoll auswählen und nutzen (Tulodziecki 1998, 11): Je nach historischer Fragestellung müssen die Lernenden entscheiden, ob eine Antwort auf ihre Fragen an die Geschichte eher in Gerichtsakten oder Zeitungen mit unterschiedlichen politischen Haltungen oder in persönlichen Briefen gefunden werden können. Durch eine gute Themen- und Quellenauswahl kann gezeigt werden, dass die Informationen nicht immer widerspruchsfrei bleiben. Beispielsweise können je nach Bestand Zeitungsausschnitte, Briefe, Tagebucheinträge und Lexikonartikel zu einem bestimmten Ereignis oder aus einer bestimmten Zeit, etwa dem 1. Weltkrieg, auf sprachlicher und besonders auch inhaltlicher Ebene verglichen werden. In der Folge kann besprochen werden, zur Beantwortung welcher Fragestellung, welches Medium geeigneter erscheint.

- Mediengestaltungen verstehen und bewerten (Tulodziecki 1998, 13): Unterschiedliche historische Darstellungen können verglichen werden und müssen in Bezug auf die Fragestellung bewertet werden. Dabei spielen sowohl formale und technische als auch die inhaltliche Gestaltung von Medien eine Rolle. Audiovisuelle Quellen müssen dabei selbstverständlich anders behandelt werden, als Zeitungen oder Zeitzeugenberichte. Insbesondere Bilder eignen sich hier für eine kontextualisierte Auseinandersetzung. Bildausschnitte in Fotografien oder die Bildkomposition in Gemälden sind selten zufällig, sollten aber bewusst reflektiert werden.

- Medieneinflüsse erkennen und aufarbeiten (Tulodziecki 1998, 14): Viele historische Ereignisse eignen sich hervorragend, um zu zeigen, welchen Einfluss die mediale Darstellung auf die weiteren Entwicklungen hatte. Als Beispiel unter vielen seien die Unruhen um die Aufführungen der 
Verfilmung «All Quiet on the Western Front» in der Zwischenkriegszeit in Deutschland und Wien genannt. Quellenmaterial dazu findet sich beispielsweise im digitalen österreichischen Zeitungsarchiv ANNO.

- Bedingungen der Medienproduktion und -verbreitung analysieren und erfassen (Tulodziecki 1998, 12-13): Zentral in der historischen Arbeit ist die Kontextualisierung einer medialen Quelle im Entstehungs-, Verbreitungs- aber auch Rezeptionszusammenhang. Anhand historischer Beispiele kann gezeigt werden, inwiefern technische, aber auch gesellschaftliche Bedingungen die Produktion und Verbreitung von Medien beeinflussen. Alleine die Konfrontation mit privaten Briefen, wie sie heute kaum noch im Umlauf sind, oder Verwaltungsakten wie Kirchenbücher, führen in ganz andere technische Medienzusammenhänge ein. Davon ausgehend können Auswirkungen dieser technischen Neuerungen, aber auch gesellschaftlicher Umstände, wie beispielsweise Bildung oder Zensur, besprochen und reflektiert werden.

Mit guter Einbindung in den schulischen Unterricht oder einer komplex gestalteten Lernumgebung lassen sich auch eigene Medienbeiträge gestalten und verbreiten (Tulodziecki 1998, 13). Ein Beispiel dazu soll am Schluss dieses Artikels stehen.

Die Bearbeitung dieser medienpädagogischen Dimensionen in einer geschichtlichen Perspektive kann auch in die Reflexion aktueller Mediendarstellungen historischer Ereignisse, wie beispielsweise Dokumentationen etc. einbezogen werden. Die bestehende historische Distanz und die bekannten beziehungsweise teilweise nachvollziehbaren historischen Auswirkungen medialer Darstellungen ermöglichen eine vertiefte Auseinandersetzung mit Berichterstattung, künstlerischen Darstellungen und gesellschaftlichen Auswirkungen, die zu einer differenzierten Reflexion aktueller Entwicklungen führen könnte. 


\section{Eine digitale Archivumgebung in der Schnittmenge der Disziplinen}

Die Schnittmengen von Archivpädagogik, Geschichtsdidaktik und Medienpädagogik erscheinen also vielversprechend für eine weitere Auseinandersetzung und nicht zuletzt als Grundlage für eine digitale Archivumgebung zu sein.

Zentral ist dabei, dass die Quellen, die Digitalisate des primären Quellenmaterials, jederzeit zugänglich sind. Eine digitale Umsetzung kann das haptische Erleben von Quellen nicht leisten, ein Archivbesuch kann also nicht gänzlich ersetzt werden. Allerdings gibt es digitale Tools, die die eigenständige Quellenarbeit von Lernenden unterstützen können. Beispielsweise kann durch halbautomatisierte Transkription alter Schriften ein unmittelbarer Zugang zu historischen Dokumenten ermöglicht werden. Auch das Überblenden von Digitalisaten, beispielsweise um Urkunden zu vergleichen, oder ein Zoom in hochaufgelöste Bilder stellt eine Chance für exploratives und forschendes Lernen dar.

Sofern ein digitales Archiv mehr ist als ein digitales Magazin, sollte Wert auf Transparenz in der Darstellung beziehungsweise Narration der Geschichte gelegt werden. Einfache Arbeitsaufträge, digitale Ausstellungen und multimediale Einstiege, die Sachwissen vermitteln, können die selbstständige Erkundung eines Archivs anregen.

Bestandteile einer digitalen Umgebung sollten also unbedingt folgende Aspekte berücksichtigen und anbieten:

- Originalquellen: Zu jeder Zeit sollte der Zugang zu den originalen Digitalisaten gewährleistet sein.

- Sachwissen und Methodenkompetenz vermitteln: Arbeitsmodule, die Sachwissen und Methodenkompetenz (Geschichtsdidaktik) aufbauen, fungieren als Basis für die Quellenarbeit. Hier wird auch vermittelt, wie gute Re-Konstruktionen entstehen.

- Transparenz in der Narration/Darstellung: Die Geschichtsdarstellungen und Narrationen, die gegebenenfalls notwendig sind, müssen als solche markiert sein und möglichst transparent formuliert sein. Texte, die von den Autorinnen und Autoren erstellt wurden, müssen sich klar von Quellenzitaten unterscheiden. 
Die pädagogische Arbeit in der Schnittmenge von Archivpädagogik, historischem Lernen und Medienbildung kann ausserdem die Re-Produktion einer eigenen Quelle oder die Gestaltung einer Geschichtsnarration unterstützen. Dabei werden nicht zuletzt zentrale historische Kompetenzen gefördert. Aber auch Ansätze zur Medienpartizipation werden adressiert. Schorb definiert Medienpartizipation als Dimension des Medienhandelns und das Vermögen «mittels Kommunikation als Austauschhandeln zwischen Menschen an der Gestaltung der menschlichen Gemeinschaft mitzuwirken bzw. an der medial gestalteten gesellschaftlichen Informations- und Kommunikationswelt zu partizipieren» (Schorb 2009, 7). Betrachtet man die unzähligen medialen Produktionen zur Geschichte, Dokumentationen, Filme und Serien mit historischen Bezug, digitale Spiele mit historischen Settings etc., die immer wieder auf sich verweisen, ist die fundierte Produktion einer Geschichtsdarstellung und die Diskussion anderer Darstellungen ein fruchtbares Feld, um Medienpartizipation zu ermöglichen.

\section{Die Gestaltung des digitalen Glasersfeld-Archivs im Rahmen des DigiVis Projektes}

Im Folgenden sollen exemplarisch die didaktischen Überlegungen, die bei der Gestaltung des Onlinezugangs zum Glasersfeld-Archiv berücksichtigt werden, erklärt werden. Die Archivumgebung soll sich in der Schnittmenge der zuvor dargestellten Bildungsbereiche bewegen und zum explorativen Lernen in einer Archivumgebung einladen.

\subsection{Grundsätzliche Überlegungen}

Das Glasersfeld-Archiv ist ein Teil des Brenner-Archivs in Innsbruck. Hier wird der Nachlass von Ernst von Glasersfeld verwaltet. Der Bestand umfasst zahlreiche wissenschaftliche Aufsätze des Philosophen, seine Arbeitsbibliothek und Fotos. Aber auch Lebensdokumente, wie ein Paar Schi oder ein selbst entworfener und gebauter Schreibtischsessel, werden hier aufbewahrt. 
Im Rahmen des DigiVis-Projektes dient das Glasersfeld-Archiv als primärer Show-Case. Ziel des Projektes ist es, generische Möglichkeiten einer digitalen Archivplattform sowie verschiedene Visualisierungszugänge aufzuzeigen und exemplarisch umzusetzen. Weitere Partnerarchive des Projektes, wie beispielsweise das Tiroler Landesarchiv weisen einen gänzlich anderen Archivbestand auf. Hier sind vor allem Dokumente aus der Landesverwaltung verwahrt. Die Tools, die das DigiVis-Projekt zu entwerfen versucht, sollen auch auf Archive angewandt werden, die eine andere Ausrichtung aufweisen.

Eine ganz zentrale Herausforderung stellt die Anonymität der Nutzer und Nutzerinnen dar. Eine Zielgruppe, die am Beginn jeder didaktischen Überlegung stehen sollte, ist nicht klar definierbar. Durch die thematische Ausrichtung des Archives lässt sich das Zielpublikum zwar etwas einschränken, dennoch bleiben wesentliche Leerstellen in der Definition.

Im Rahmen des Projektes haben wir uns daher entschieden von Use Cases, also unterschiedlichen Nutzungsszenarien einer Archivseite, auszugehen: Einzelne Besucherinnen- und Besuchergruppen stellen gewisse Erwartungshaltungen an ein Archiv. Wollen Forscherinnen und Forscher möglichst nahe an die originale Quelle und sind das Arbeiten mit Suchfeldern etc. gewöhnt, suchen Lehrkräfte nach Material für bestimmte Themen im Lehrplan und Lernende sowie Interessierte benötigen einen intuitiven Einstieg in die Themen des Archivs.

Ziel ist es, für jedes Publikum mögliche Anknüpfungspunkte zu schaffen, die sie immer weiter ins Archiv begleiten, bis schliesslich eine eigenständige Quellenarbeit unterstützt wird.

Dazu sollen durch didaktische Bausteine einfache Einstiege in das Thema des Archives ermöglicht werden. Diese Bausteine arbeiten zum Teil mit den Inhalten aus dem Archiv, aber auch mit originalen Digitalisaten. Die Einleitungen zum Thema sind als Darstellungen der Geschichte beziehungsweise der Inhalte zu verstehen und müssen auch als solche gekennzeichnet werden. Für den User muss zu jeder Zeit klar sein, ob er mit authentischen Dokumenten aus der Geschichte beziehungsweise Texten, die Glasersfeld selbst schrieb, arbeitet, oder aber mit kuratierten Inhalten, die zur Einleitung in das Archiv gestaltet wurden, konfrontiert ist. Darüber hinaus sind diese didaktischen Bausteine an Lehrplaninhalte 
unterschiedlicher Fächer angelehnt, die für Lehrende auch nachvollziehbar gemacht werden. Ein Baustein soll nicht über den Arbeitsaufwand einer Unterrichtsstunde hinausgehen, um den Lehrkräften Freiheiten in der Vor- und Nachbereitung zu ermöglichen und die Aufmerksamkeit der Nutzerinnen und Nutzer zu behalten. Eine Kombination einzelner Bausteine ist allerdings möglich. Bisher angedachte Bausteine beschäftigen sich mit Glasersfelds Verständnis von Sprache (Deutsch und Philosophie), dem Konzept der Viabilität (interaktives Memoryspiel) oder unterschiedlichen Definitionen von Identität (interaktives Video). Diese Bausteine sollen als Unterrichtseinstieg, Erarbeitungsphase oder Wiederholung des Gelernten, aber auch zur eigenständigen Erkundung nutzbar sein.

Ausserdem sollen, sobald die angedachten Visualisierungen und die Forschungswerkzeuge, wie Named entity recognition etc. implementiert sind, auch Bausteine mit methodischen Inhalten entstehen.

Ziel der einführenden Angebote ist es, Lernende zu befähigen, sich inhaltlich und methodisch so weit einzuarbeiten, dass eine eigenständige Arbeit mit den digitalisierten Archivbeständen möglich ist und die Nutzerinnen und Nutzer fähig sind, die Tools, die auf der Archivoberfläche verfügbar sind, zu bedienen.

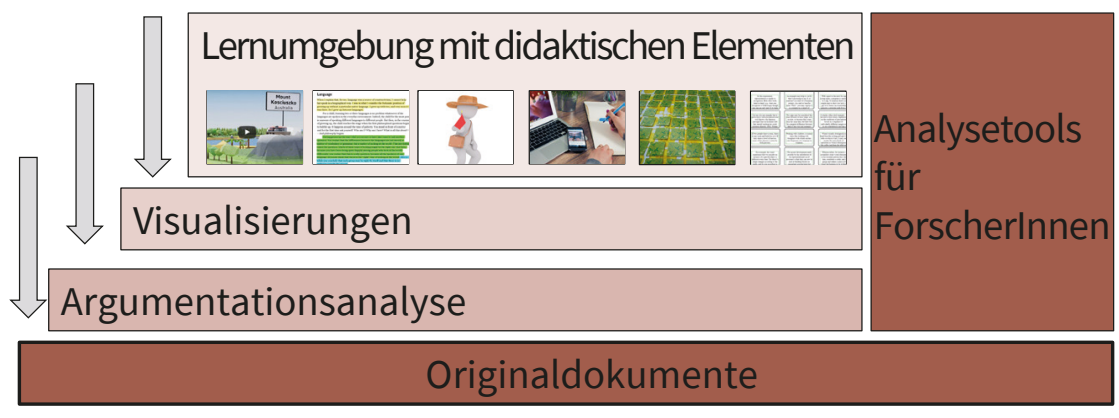

Abb. 4.: Schematische Darstellung des didaktischen Grundkonzepts der digitalen Lernumgebung im Glasersfeld-Archiv. Die Pfeile symbolisieren ein immer tieferes Eindringen in die Thematik bis hin zu den Originaldokumenten, die jederzeit zugänglich sind. 


\subsection{Beispiel für ein didaktisches Modul an der Schnittstelle von historischem Lernen und Medienpädagogik}

Ein digitales Tool, das für die Einführung aber auch für die vertiefende Auseinandersetzung mit Quellen und Thematiken geeignet ist, ist das Erstellen von «Spaziergängen» durch die Sammlung.

In der Rezeption von Spaziergängen soll ein einfaches und begleitetes Kennenlernen und Eintauchen in den Archivbestand möglich sein. Dabei ist es wichtig, dass immer der Zugang zu den Originalquellen möglich ist und Transparenz über die Darstellung der Quellen angestrebt wird.

Gleichzeitig soll aber auch die Produktion solcher Spaziergänge ermöglicht werden:

Auf einem einfachen und intuitiv bedienbaren Interface werden Quellen, Textausschnitte und/oder Digitalisate zur Auswahl gestellt. Diese Darstellung kann durchaus an einem Generous interface orientiert sein. Anhand von thematischen, zeitlichen oder materiellen Kategorien kann der User die Auswahl der angezeigten Artefakte filtern. Interessante Objekte können in die individuelle Auswahl für die Ausstellung gezogen werden.

Abb. 5 zeigt exemplarisch das Interface für Texte aus dem GlasersfeldArchiv. Die Textstellen stammen aus der Argumentationsanalyse von Primärtexten aus Glasersfelds Nachlass. Die Textausschnitte lassen sich nach Themen, aber auch nach argumentativen Kategorien wie Narrativ, Beispiel oder Prämisse sortieren und filtern. Über das Textfeld ist jeweils auch der Originaltext zugänglich. 


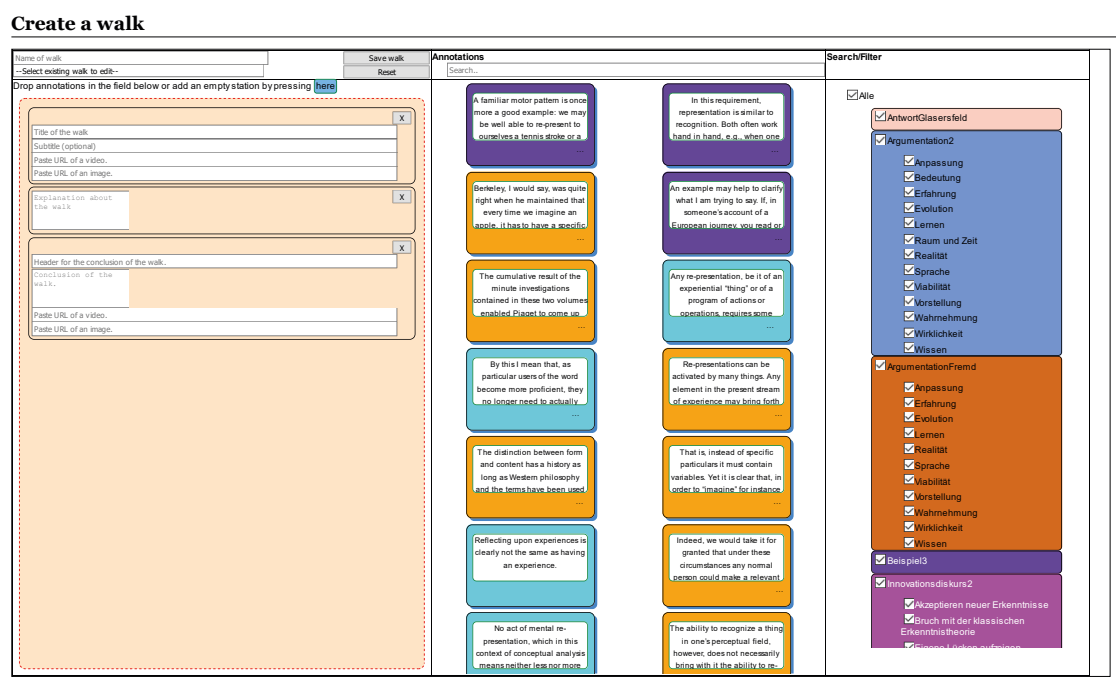

Abb. 5.: Screenshot des Work-in-Progress-Interfaces zur Erstellung eigener Spaziergänge durch das digitalisierte Archivmaterial (https://dbis-digivis. uibk.ac.at/mediawiki/index.php/Special:WalkCreatorDigiVis).

In einem nächsten Schritt erstellt die Userin einen Spaziergang, in dem sie ihre persönliche Auswahl in einen narrativen Darstellungszusammenhang bringt. Möchte sie beispielsweise eine Ausstellung zum Thema Wahrheit gestalten, kann sie anhand von Textstellen, die sie in einen logischen Zusammenhang stellt anhand von Glasersfelds Beispielen, Narrativen, die er in seinem Werk verwendet oder Argumenten den eigenen Zugang zu Wahrheit darstellen.

Andere Userinnen und User sollen diesem Zugang später über die Archivseite folgen und ihn kommentieren können. Ob die Userin im Archiv arbeitet, als Lehrerin ein Modul für den eigenen Unterricht erstellt, als Studentin einer Arbeitsaufgabe nachkommt oder als Interessierte ihren eigenen Zugang zur Diskussion stellen möchte, ist dabei nebensächlich.

Ein solcher Zugang zu philosophischen Texten braucht sicher einiges an Vorwissen, ist die Quellenlage aber eine andere, sind solche Zugänge auch mit jüngeren Schülerinnen und Schülern oder weniger eingearbeiteten interessierten Nutzerinnen und Nutzern denkbar:

Beispielsweise könnte anhand von Kirchenbüchern und Grundbuchblättern aus dem Tiroler Landesarchiv eine Familiengeschichte dargestellt werden. Die Quellen gelten darin als Beleg für historische Ereignisse, die 
durch einen erklärenden Text verbunden werden müssen. Dabei fallen auch die Leerstellen auf, die in unterschiedlichen historischen Epochen bei unterschiedlichen historischen Persönlichkeiten verschieden gross sind.

Durch das eigenständige Durchsuchen und Erforschen der Sammlung können eng verbunden mit Quellenmaterial eigene Ausstellungsnarrationen entstehen. Durch die Kommentarfunktion, die eine Diskussion der Darstellungen ermöglicht, können alle drei behandelten pädagogischen beziehungsweise didaktischen Zugänge vereint werden: Die Archivseite wird zum Raum, in dem Geschichte anhand von Quellen erkundet werden kann, Narrationen und Geschichtsvorstellungen können anhand dieser dargestellt werden und schliesslich wird eine Diskussion und Reflexion dieser Darstellungen in einem sozialen Austausch angeregt. So ist es je nach späteren Betreuungsmöglichkeiten des Archivs möglich, öffentlich Kommentare zu moderieren oder durch individuelle Zugänge für Schulklassen einen Austausch innerhalb einer Lernendengruppe zuzulassen. Es werden Kompetenzen der Quellenarbeit gefördert, die Reflexion von Geschichtsbildern und medialen Darstellungen angestossen und der einzelne User tritt in ein mediales (Ver-)Handeln seiner historischen Erzählung. Ein derartiger Zugang scheint nicht nur für den schulischen, aber auch universitären Unterricht interessant, sondern auch für interessierte «Flaneure» (vgl. Grünberger 2014), die sich auf Archivseiten erkunden und Eindrücke sammeln, oder aber möglicherweise auch ihre eigene Perspektive in einer Ausstellung veröffentlichen wollen.

Das Rijksmuseum bietet bereits die Möglichkeit eine eigene Sammlung zu gestalten an (Rijksmuseum). Im Gegensatz zu den Spaziergängen fehlt eine Kommentarfunktion, besonders aber die fehlende Kontextualisierung, Verbindung und Argumentation der gesammelten Werke regen eine Reflexion der eigenen Auswahl allerdings nicht an.

\section{Generische Zusammenfassung und Ausblick}

Die zunehmende Digitalisierung von kulturellem Wissen durch Digitalisierungsbemühungen, aber auch die zunehmend anfallenden digitalen Artefakte, machen die Frage, wie Archive zukünftig zugänglich sein sollen, dringlich. Das umfangreiche Potential von digitalen Archiven 
als Informations- und Lernräume, die nicht zuletzt auch Flaneure (vgl. Grünberger 2014) zum Verweilen und Erkunden anregen, wird bislang noch nicht ausgeschöpft.

Die Schnittmenge von Archivpädagogik, Geschichtsdidaktik und Medienbildung scheint vielversprechend für die Gestaltung attraktiver und vielfältig nutzbarer Zugänge zu digitalem Archivmaterial zu sein. Zentral ist dabei die Arbeit mit digitalisierten Quellen, die als Zeugnisse der Geschichte als Grundlage für Geschichtsnarrationen und -darstellungen dienen.

Ziel der pädagogischen Arbeit im Archiv ist die Befähigung zur selbstständigen Arbeit mit historischen Quellen unterschiedlicher Art. Daher erscheinen besonders Zugänge zu Archiven interessant, in denen nach einer kurzen Einarbeitung in den fachlichen Kontext des Archives und einfache digitale Werkzeuge selbstständiges Arbeiten mit digitalisierten Quellen möglich ist. Diese Arbeit kann mehr oder weniger frei passieren und in unterschiedlichen Bildungsprozessen innerhalb und ausserhalb von Schulen und Archiven eine Rolle spielen. Über digitale Medien erhält das «Verhandeln» von Geschichte eine neue Dimension, die durch derartige Zugänge nutzbar gemacht werden kann.

Geschichtsnarrationen, die im Rahmen der Spaziergänge, die als Beispiel vorgestellt wurden, entstehen, tragen enormes Reflexionspotential in sich. Die Darstellung zweier Mitschüler zur selben Aufgabe kann sich nämlich je nach Quellenlage stark unterscheiden. Unterschiedliche Perspektiven aus unterschiedlichen Altersgruppen oder Kulturen, auch über Landesgrenzen und nationale Geschichtsschreibung hinaus, können sichtbar und diskutierbar gemacht werden.

Dieser Beitrag zeigt, dass in der Schnittmenge von Medienbildung, Geschichtsdidaktik und Archivpädagogik ein enormes Bildungspotential gefunden werden kann, das nicht zuletzt in digitalen Archivzugängen zunehmend genutzt werden sollte. 


\section{Literatur}

Anno Historische Zeitungen und Zeitschriften. o.J. "Anno-Suche Volltextsuche in ausgewählten Zeitungen». http://anno.onb.ac.at/suchhilfe.htm\#chapl.1.

Archivschule Marburg. o.J. «Archivwissenschaftliche Kolloquien». https://www. archivschule.de/DE/forschung/archivwissenschaftliche-kolloquien/.

Aspelmeier, Jens. 2019. «Geschichte selber erkunden》-Geschichtsdidaktische Überlegungen zu Chancen und Grenzen Historischen Lernens im und mit dem Archiv». Archivar. Zeitschrift für Archivwesen Neue Tendenzen in der Archivpädagogik (2): 105-8. http://www.archive.nrw.de/archivar/hefte/2019/Ausgabe-2/Archivar-2-2019.pdf.

Bernsen, Daniel. 2017. «Medien im Geschichtsunterricht: Funktionen, Verhältnis und Raumverständnis». In Praxishandbuch Historisches Lernen und Medienbildung im digitalen Zeitalter, herausgegeben von Daniel Bernsen und Ulf Kerber, 37-44. Opladen Berlin Toronto: Verlag Barbara Budrich.

Bernsen, Daniel, und Ulf Kerber, Hrsg. 2017. Praxishandbuch Historisches Lernen und Medienbildung im digitalen Zeitalter. Opladen Berlin Toronto: Verlag Barbara Budrich.

Bounin, Ingrid. 2015. «Medienbildung in Baden-Württemberg». LMZ Mediaculture online (blog). 2015. https://web.archive.org/web/20150405054510/http://www. lmz-bw.de/medienbildung-baden-wuerttemberg.html.

Bundesministerium für Digitalisierung und Wirtschaftsstandort. 2000. Bundesgesetz über die Sicherung, Aufbewahrung und Nutzung von Archivgut des Bundes (Bundesarchivgesetz). https://www.ris.bka.gv.at/GeltendeFassung.wxe?Abfrag $\mathrm{e}=$ Bundesnormen\&Gesetzesnummer $=10010143$.

Diener Steackling, Antje. 2018. «Wie entwickle ich eine Social-Media-Strategie für mein Archiv? - Oder: Die optimale Kosten-Nutzen-Rechnung.» Archivar. Zeitschrift für Archivwesen Archive im Web 2.0 (1): 28-32.

Drucker, Johanna. 2013. «Performative Materiality and Theoretical Approaches to Interface». digital humanities quarterly (blog). 2013. http://www.digitalhumanities.org/dhq/vol/7/1/000143/000143.html.

«Europeana». o.J. Europeana (blog). https://www.europeana.eu/portal/de.

Glaunert, Mario. 2015. «Archivbenutzung im Digitalen Zeitalter.» In Brandenburgische Archive. Berichte und Mitteilungen aus den Archiven des Landes Brandenburg. Brandenburgische Archive. Berichte und Mitteilungen zu den Archiven des Landes Brandenburg 33. https://blha.brandenburg.de/wp-content/uploads/2017/10/Brandenburgische_Archive_33_2016.pdf.

Grünberger, Nina. 2014. «Räume zum Flanieren, Spielen und Lernen. Überlegungen zur Gestaltung von Bildungs- und Lernräumen im Kontext kultureller Entwicklungen.»In Lernräume gestalten - Bildungskontexte vielfältig denken, herausgegeben von Klaus Rummler, 56-67. Medien in der Wissenschaft 67. Münster: Waxmann.

Kan, Fred van. 2018. «Vollständige Online-Dienste. Möglichkeiten und Unmöglichkeiten». Archivar. Zeitschrift für Archivwesen Archive im Web 2.0 (1): 25-28. 
Kerres, Michael. 2018. Mediendidaktik: Konzeption und Entwicklung digitaler Lernangebote. Fifth edition. De Gruyter Studium. Boston, Massachusetts: Walter de Gruyter GmbH.

Lange, Thomas, Thomas Lux, und Ulrich Mayer. 2004. Historisches Lernen im Archiv. Methoden Historischen Lernens. Schwalbach/Ts: Wochenschau-Verl.

Näpel, Oliver. 2015. «Medienbildung im Geschichtsunterricht I: Geschichte und digitale Medien». LMZ Mediaculture online (blog). 2015. https://web.archive.org/web/20150405143114/https://www.lmz-bw.de/medienbildung-geschichtsunterricht-1.html.

Odendahl, Tim. 2018. "Archive im Quadrat. Instagram für Archive». Archivar. Zeitschrift für Archivwesen Archive im Web 2.0 (1): 44-47.

Rönz, Andrea. 2018. "Social Media in deutschsprachiven Archiven - der momentane Stand der Dinge». Archivar. Zeitschrift für Archivwesen Archive im Web $2.0(1): 41-44$.

Schaller, Annekatrin. 2019. "Anspruch und Wirklichkeit. Archivpädagogik in Deutschland heute». Archivar. Zeitschrift für Archivwesen Neue Tendenzen in der Archivpädagogik (2): 102-5. http://www.archive.nrw.de/archivar/hefte/2019/Ausgabe-2/Archivar-2-2019.pdf.

Schorb, Bernd. 2009. «Gebildet und kompetenz. Medienbildung statt Medienkompetenz?» merz. Medien+Erziehung. Zeitschrift für Medienpädagogik, Nr. 53. Jahrgang, Nr. 5 (Oktober): 50-56. https://www.lmz-bw.de/fileadmin/user_upload/ Downloads/Handouts/schorb-gebildet-und-kompetent.pdf.

Stüber, Gabriele. 2003. "Qualitätsparameter archivischer Arbeit - Überlegungen zur Dienstleistung und Ressourcengewinnung». Der Archivar. Mittelungsblatt für deutsches Archivwesen., 56 (3): 203-13. http://www.archive.nrw.de/archivar/hefte/2003/Archivar_2003-3.pdf.

Theimer, Kate. 2018. «Partizipation als Zukunft der Archive». Archivar. Zeitschrift für Archivwesen Archive im Web 2.0 (1): 6-13.

Tulodziecki, Gerhard. 1998. «Entwicklung von Medeinkompetenz als Erziehungsund Bildungsaufgabe.» Pädagogische Rundschau, Nr. 52 (1998) 6: 693-709. https://nbn-resolving.org/urn:nbn:de:Oll1-opus-14821.

Unger, Thorsten. 2018. «Archivisches Bloggen jenseits des (Mainstream)? Die Blogs des Universitätsarchivs und des Historischen Vereins Osnabrück». Archivar. Zeitschrift für Archivwesen Archive im Web 2.0 (1): 32-37.

Whitelaw, Mitchell. 2013. «Towards Generous Interfaces for Archival Collections», ICA Congress Brisbane, . http://mtchl.net/assets/Whitelaw_ICA_GenerousInterfaces.pdf.

Whitelaw, Mitchell. 2015. «Generous Interfaces for Digital Cultural Collections». DHQ: Digital Humanities Quarterly 9 (1). http://www.digitalhumanities.org/ $\mathrm{dhq} / \mathrm{vol} / \mathrm{g} / \mathrm{I} / 000205 / 000205 \cdot \mathrm{html}$.

Whitelaw, Mitchell. o.J. «Exploring the Manly Local Studies Image Library». Manly Images (blog). http://mtchl.net/manlyimages/index.html.

Wolf, Thomas. 2018. «Blogs in deutschen Archiven. Ein kommentierter Überblick». Archivar. Zeitschrift für Archivwesen Archive im Web 2.0 (1): 37-41. 